\title{
Caveolae Depletion Contributes to Vasorelaxant Effects of Chenodeoxycholic Acid
}

\author{
Zhongchao Wanga,c Qiang Lv ${ }^{\mathrm{a}}$ Huan Liu ${ }^{\mathrm{a}}$ Yue Wu $\mathrm{W}^{\mathrm{d}}$ Yungang Baia \\ Yaoping Cheng ${ }^{b}$ Yuting Sub Yue Cai ${ }^{a}$ Jinwen Yua Jin Maa Junxiang Bao ${ }^{a, b}$ \\ aDepartment of Aerospace Physiology, Fourth Military Medical University, Xi'an, bepartment of \\ Aerospace Hygiene, Fourth Military Medical University, Xi'an, 'Department of Congenital Heart \\ Disease, PLA Shenyang General Hospital, Shenyang, dDepartment of Neurology, PLA Shenyang General \\ Hospital, Shenyang, P. R. China
}

\section{Key Words}

Caveolae $\cdot$ Chenodeoxycholic acid $\cdot$ Cholic acid $\cdot$ Methyl- $\beta$-cyclodextrin $\bullet$ Vasorelaxant effect - Endothelial nitric oxide synthase $\cdot$ Nitric oxide

\begin{abstract}
Background/Aims: High concentration of bile acids (BAs) induces hydrophobicity-dependent vasorelaxtant effects with hydrophobic BAs showing greater responses than hydrophilic BAs, of which the underlying mechanisms are still unclear. Caveolae are invaginations on membranes of endothelial cells (ECs) entraping endothelial nitric oxide synthase (eNOS) to prevent its activation, which plays a critical role in regulation of vascular function. The purpose of the present study was to investigate the role of caveolae in vasorelaxant effects of BAs. Methods: Chenodeoxycholic acid (CDCA) and cholic acid (CA) were used to represent hydrophobic and hydrophilic BA, respectively. Vascular responses of abdominal aorta were measured by isometric force recording. Morphology of caveolae was examined by transmission electron microscopy. Protein expression of total eNOS (t-eNOS) or phosphorylated eNOS ( $p$-eNOS) was determined by Western blot. Nitric oxide (NO) content was observed by fluorometric assay. Results: We demonstrated that CDCA as well as Methyl- $\beta$-cyclodextrin (MCD), a commonly used reagent for cholesterol depletion, reduced potassium chloride (KCl)- or phenylephrine (PE)elicited vasoconstriction $(P<0.05)$, and enhanced acetylcholine (Ach)-elicited vasodilatation $(P<0.05)$ in endothelium-intact abdominal aorta but not in endothelium-denuded or CAtreated vessels. CDCA and MCD, but not CA significantly disrupted caveolae structure on ECs of abdominal aorta which was recovered by cholesterol incubation $(P<0.05)$. Protein expression of t-eNOS was significantly decreased $(P<0.05)$, and that of $\mathrm{p}$-eNOS together with NO content was significantly increased in CDCA- and MCD- but not CA-treated vessels $(P<$ 0.05 ) as compared with vehicle. The effect was reversed by either endothelium-denudation or cholesterol replenishment. Moreover, with cholesterol incubation, no significant differences were found in vascular responses among CDCA-, CA- or vehicle-treated vessels. Conclusion:
\end{abstract}

Z. Wang, Q. Lv and H. Liu contributed equally to the work.

Junxiang Bao, M.D. Ph.D.

and Jin Ma, M.D., Ph.D.

KARGER
Department of Aerospace Hygiene, Fourth Military Medical University, Xi'an 710032

(P. R. China); Department of Aerospace Physiology, Fourth Military Medical University,

Xi'an 710032, (P. R. China)

E-Mail baojunx@fmmu.edu.cn / jin-ma@fmmu.edu.cn 
These results indicate that CDCA diminishes caveolae on ECs of abdominal aorta promoting eNOS phosphorylation and NO production which contributes to its vasorelaxtant effect.

\section{Introduction}

(C) 2017 The Author(s)

Published by S. Karger AG, Basel

Bile acids (BAs) are products of cholesterol metabolism and facilitate absorption of dietary lipids. They are produced in the liver and released into small intestine where most of them are reabsorbed into portal vein for being released again by hepatocytes $[1,2]$. The whole process is named as enterohepatic circulation which entails the efficient usage of BAs and keeps the concentration of BAs in systemic circulation under $10 \mu \mathrm{mol} / \mathrm{L}$ [3]. However, in hepatic diseases exhibiting cholestasis, the serum concentration of BAs could rise sharply even reaching $200 \mu \mathrm{mol} / \mathrm{L}$ and act as a strong stimulus to the structural or functional remodeling of cardiovascular system [4].

Over the past decade, emerging evidences have indicated high dose of BAs as vasorelaxants which partly accounts for the systemic hypotension developed with the cholestasis [5]. Moreover, the potency of BA-induced vasorelaxation depends much on the lipid solubility. Normally, hydrophobic BAs exhibit greater effect than hydrophilic ones, underlying which multiple mechanisms have been proposed [6]. For example, deoxycholic acid (DCA) or chenodeoxycholic acid (CDCA) prevents activity of contractile surface membrane receptors through lipid peroxidation [6]. CDCA promotes nitric oxide (NO) production in vascular endothelial cells (ECs) by interaction with TGR5, a plasma membrane receptor [7]. In addition, lithocholaste activates large $\mathrm{Ca}^{2+}$-activated $\mathrm{K}^{+}$channels $\left(\mathrm{BK}_{\mathrm{Ca}}\right)$ to dilate small arteries [8]. Nevertheless, how the BAs induce vasorelaxation is still not fully understood.

Caveolae are membrane invaginations abundant in vascular cells which are 50-100 nm in size and enriched of cholesterol and lipids with saturated acyl chains, such as sphingolipids and glycosphingolipids [9]. The involvement of caveolae in vesicular transportation, cholesterol homeostasis, signal transduction and mechanosensing has been recognized for a long time [10]. Besides, caveolae act as a platform where numerous lipid-modified signaling molecules such as Src-family kinases, Ras GTPases, G-proteins, glycosyl-phosphatidylinositol (GPI)-linked proteins are located, so the integrity of caveolae is critical for the activation of these molecules [10]. To be emphasized, in ECs, endothelial nitric oxide synthase (eNOS) is normally restricted to caveolae where it resides in a signaling module by binding to caveolin-1 (Cav-1), the main structural protein of caveolae, thus its phosphorylation as well as NO production is hindered $[11,12]$.

The formation of caveolae largely depends on cellular cholesterol metabolism, so modification of cholesterol content is a common way to adjust the quantity of caveolae on cell membrane [13]. It is well acknowledged that BAs can solubilize membranes extracellularly by promoting cholesterol and phospholipid clearance $[6,14]$. So we hypothesized that BAs disturbed caveolae intactness on ECs membranes through diminishing cholesterol which induced vasorelaxation by facilitating eNOS phosphorylation and NO production.

Both CDCA and cholic acid (CA) are primary BAs with CDCA owning one less hydroxyl group than CA which makes it more lipophilic [6]. In this study, CDCA and CA were adopted to represent hydrophobic and hydrophilic BA, respectively, and the effects of these two BAs on caveolae abundance, eNOS phosphorylation, NO production and vascular responses were analyzed and compared.

\section{Materials and Methods}

Ethical Approval

All experimental treatments and procedures employed in this study were approved by the Institutional Animal Care and Use Committee of the Fourth Military Medical University, and in accordance with the rules 


\section{Cellular Physiology Cell Physiol Biochem 2017;42:1013-1024 \begin{tabular}{l|l} 
and Biochemistry Published ondine: June 28, 2017 & $\begin{array}{l}\text { (c) } 2017 \text { The Author(s). Published by S. Karger AG, Basel } \\
\text { www.karger.com/cpb }\end{array}$
\end{tabular}}

Wang et al.: Caveolae Counteracts Vasorelaxant Effects of CDCA

and regulations described in the United States National Institutes of Health Guide for the Care and Use of Laboratory Animals.

\section{Animals and Vascular Tissue Preparations}

Animals were supplied by the Laboratory Animal Centre of the Fourth Military Medical University. Male Sprague-Dawley rats, aged 7-9 wk and weighing between 260-280 g were individually caged within a $12 \mathrm{~h}: 12 \mathrm{~h} \mathrm{light/dark}$ cycle at $23 \pm 1^{\circ} \mathrm{C}$ with standard lab chow and water available ad libitum. After one week of acclimatization, rats were randomly assigned to 8 groups ( $\mathrm{n}=12$ /group), including vehicle (Vehl), CDCA, Methyl- $\beta$-cyclodextrin (MCD), CA, Vehl + cholesterol (Chl), CDCA + Chl, MCD + Chl and CA + Chl group.

After preparations, rats were weighed and anesthetized by intraperitoneal injection of pentobarbital sodium $(40 \mathrm{mg} / \mathrm{kg}$ ) and killed by exsanguination via the heart. Then the abdominal aorta were rapidly removed and placed in pre-cold Krebs buffer solution consisting of (in mM): $118.3 \mathrm{NaCl}, 4.7 \mathrm{KCl}, 25$ $\mathrm{NaHCO}_{3}, 1.2 \mathrm{MgSO}_{4}, 1.2 \mathrm{KH}_{2} \mathrm{PO}_{4}, 2.5 \mathrm{CaCl}_{2}, 11.1$ glucose, and 0.026 EDTA (pH 7.4). Afterwards, vessels went through careful dissection under a microscope. Intact artery rings were then prepared for the isometric force measurement and the remains were snap frozen in liquid nitrogen and kept at $-80^{\circ} \mathrm{C}$ for Western blot analysis or homogenated immediately for total nitric oxide (NO) content measurement.

\section{Isometric Force Measurement}

To exclude the potential confounding effects from myogenic tone, we used in vitro measurement of isometric force to examine the vasoreactivity of abdominal aorta, which was conducted according to the methods described by Wang et al. [15]. Abdominal aortic rings $3 \mathrm{~mm}$ in length were made and mounted onto two gracile stainless steel hooks and suspended in the four-chamber organ bath (Radnoti, USA), with one hook attached to the plastic holder in the chamber, and the other to the tension transducer connected to Powerlab (ADInstruments, Bella Vista NSW, Australia) for vasoreactivity recording. The chamber was filled with Krebs buffer solution, kept at $37^{\circ} \mathrm{C}$ and bubbled continuously with $95 \% \mathrm{O}_{2} \sim 5 \% \mathrm{CO}_{2}$. After equilibration for $30 \mathrm{~min}$, rings were stimulated by $60 \mathrm{mM}$ potassium chloride $(\mathrm{KCl})$ and washed thoroughly back to the initial resting force repeatedly till the optimal resting tension was achieved. Subsequently, following preincubation with CDCA $\left(10^{-4} \mathrm{M}\right)$, CA $\left(10^{-4} \mathrm{M}\right)$, Chl $(10 \mathrm{mM})$ or MCD $(10 \mathrm{mM})$ for $1 \mathrm{~h}$, the artery rings were pre-contracted by $10^{-6} \mathrm{M}$ phenylephrine (PE), and then concentration response curves elicited by 10 100 $\mathrm{mM} \mathrm{KCl}, 10^{-10} \sim 10^{-4} \mathrm{M}$ PE, acetylcholine (Ach) and sodium nitroprusside (SNP) were examined. In another protocol, endothelium of the artery rings was removed by inserting a stainless steel needle into the lumen rubbing the inner face slightly followed by accumulative concentration-dependent vasoactive responses. Concentration response curves were analyzed using an agonist-response best-fit line. The vasodilative responses were expressed as percentage of the contraction elicited by PE. The overall vasoconstrictive or vasodilative capacity was expressed as area under curves (AUC).

\section{Transmission Electron Microscope (TEM)}

Following deep anesthetization, rats were perfused with PBS (pH 7.4) and then with pre-cold 4\% formaldehyde and $2.5 \%$ glutaraldehyde in PBS. Abdominal aorta were rapidly removed and carefully dissected as described above. Subsequently, with or without pretreatment by CDCA, CA, Chl or MCD at 37 ${ }^{\circ} \mathrm{C}$ for $1 \mathrm{~h}$, artery rings were prepared and kept in $2.5 \%$ glutaraldehyde in PBS (pH 7.4). Then the rings were further fixed in 1\% osmium acid for $1 \mathrm{~h}$. Afterwards, rings went through dehydration in graded ethanol, followed by dry acetone and finally embedded in epoxyresin. Ultrathin sections $(100 \mathrm{~nm})$ were obtained and viewed under a JEM-2000EX TEM (JEOL, Tokyo, Japan) to find the characteristic structure of caveolae, which were distinguished by their size (50-100 nm), flask-like shape, and location on or juxtaposition to the plasma membrane [9]. Two observers blind about the aim of the experiment counted the caveolae number in randomly selected 20 fields separately and then made the average to get the caveolae number on each field of the vessel.

\section{Western Blot}

Homogenization and Western blot analysis were performed as previously described to identify the endothelial nitric oxide synthase (eNOS) protein abundance and phosphorylation state at $\mathrm{Thr}^{495}$. Briefly, vessels were minced and homogenized in tissue protein extraction reagent (Pierce, Rockford, IL, USA) containing protease inhibitor (Pierce, Rockford, IL, USA) and phosphotase inhibitor (Roche, Basel, 


\section{Cellular Physiology Cell Physiol Biochem 2017;42:1013-1024

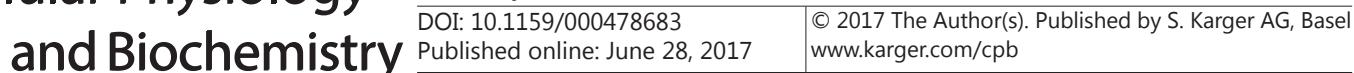

Wang et al.: Caveolae Counteracts Vasorelaxant Effects of CDCA

Switzerland) with the aid of a glass tissue grinder. Lysates were then centrifuged at 12, $000 \mathrm{~g}$ for $10 \mathrm{~min}$ at $4^{\circ} \mathrm{C}$ and the supernatants were collected. Protein concentrations were determined with a bicinchoninic acid assay kit (Pierce, Rockford, IL, USA) and equivalent amount of proteins were loaded onto 4-12\% Bis-Tris PAGE gels within the NuPAGEBis-Tris Pre-cast Gel System (Invitrogen Life Technologies, Carlsbad, CA, USA). Afterwards, the fractionated proteins were electrophoretically transferred to polyvinylidene difluoride (PVDF) membranes (Millipore, Billerica, MA, USA) in an XCell Blot Module transfer system (Invitrogen Life Technologies, Carlsbad, CA, USA). Membranes were then blocked for $6 \mathrm{~h}$ at room temperature by $5 \%$ BSA in TBS containing $0.1 \%$ (wt/vol) Tween 20 (TBS-T). Subsequently, membranes were incubated with mouse monoclonal antibody against eNOS $(1: 1,000$, Cell Signaling Technology, Boston, MA, USA) or phospho-eNOS ( $\mathrm{Thr}^{495}, 1: 1,000$, Cell Signaling Technology, Boston, MA, USA) in TBS-T at $4^{\circ} \mathrm{C}$ overnight, respectively. After incubation, membranes were washed with TBS-T and went through another incubation with horseradish peroxidase conjugated goat anti-mouse IgG (1:12, 000, Proteintech, Chicago, IL, USA) for 90 min at room temperature. The bound antibodies were detected by enhanced chemiluminescence detection reagents (Millipore, Billerica, MA, USA) in Gel Image Analyzing System (Tanon-4200, Tanon Science \& Technology, Shanghai, China). Densitometry analysis of bands was performed by ImageJ software (National Institutes of health, USA).

Nitric Oxide (NO) Assay

We applied the Total Nitric Oxide Assay Kit (Beyotime Institute of biotechnology, Shanghai, China) to measure NO content in abdominal aorta under several certain conditions. Experiments were conducted in accordance with the instruction of the kit. In brief, vessels were collected and incubated with CDCA, CA, Chl or MCD for $1 \mathrm{~h}$ in Krebs buffer solution at $37^{\circ} \mathrm{C}$. Then tissues were minced and homogenized with the recommended tissue protein extraction reagent (Beyotime Institute of biotechnology, Shanghai, China). Following centrifugation, supernatants were collected and protein concentrations were measured. Equivalent amount of proteins were then loaded into 96-well plate, in addition with a series of reagents listed in the instruction. Since NO is not stable and could be rapidly processed into nitrate and nitrite by cellular metabolism, we could estimate the total NO content through measuring the amount of nitrate and nitrite. A spectrophotometer was used to read the absorbance at $540 \mathrm{~nm}$ as the relative nitrate and nitrite content. Standard curve was also performed and the absolute NO content was thus calculated.

\section{Data Analysis}

Data are expressed as means \pm SE. Student's t-test was used to determine the significance of differences in protein expression, caveolae number and NO content between groups. Isometric force measurements were analyzed by repeated-measures two-way ANOVA. Statistical analysis was performed using SPSS 16.0 (SPSS Inc) software and Prism software 5.0 (Graphpad). A value of $P<0.05$ was considered to be statistically significant.

\section{Results}

Effects of CDCA or CA on vasoreactivity of abdominal aorta

Vasorelaxtant activity of BAs might affect both vasoconstriction and vasodilatation, so we first examined the effects of CDCA or CA on vasoactive responses of abdominal aorta to $\mathrm{KCl}$ or PE which contracts arterial smooth muscle by reducing membrane potential or activating adrenoceptors. Then, two kinds of vasodilators were also employed, including Ach and SNP, which elicit endothelium-dependent and -independent vasodilatation, respectively. AUC was calculated to represent the overall vasoconstrictive or vasodilative capacity of abdominal aorta. To explore the role of caveolae in BAs-elicited vasorelaxation, we also compared the vasoactive effect of BAs with that of MCD, a commonly used reagent for Chl depletion which could remove caveolae structure efficiently. As shown by Fig. 1A and B, compared with Vehl, CDCA and MCD but not CA significantly reduced the vasoconstrictive responses elicited by either $\mathrm{KCl}$ or PE $(P<0.05)$. In addition, CDCA and MCD, but not CA significantly enhanced the endothelium-dependent vasodilative response elicited by Ach (Fig. 1C, $P<0.05$ ). The SNP-elicited endothelium-independent vasodilatation was not affected by CDCA, MCD or CA 
Fig. 1. Effects of chenodeoxycholic acid (CDCA), cholic acid (CA) or Methyl- $\beta$-cyclodextrin (MCD) on vascular responses of abdominal aorta. Concentration-dependent responses of endothelium-intact abdominal aorta to potassium chloride ( $\mathrm{KCl}, \mathrm{A})$, phenylephrine (PE, B), acetylcholine (Ach, C) or sodium nitroprusside (SNP, D) in the presence of vehicle (Vehl), CDCA, CA or MCD and the area under curves (AUC) of each test. Values are means \pm SE. $n=8,{ }^{*} P<0.05$ vs. Vehl.

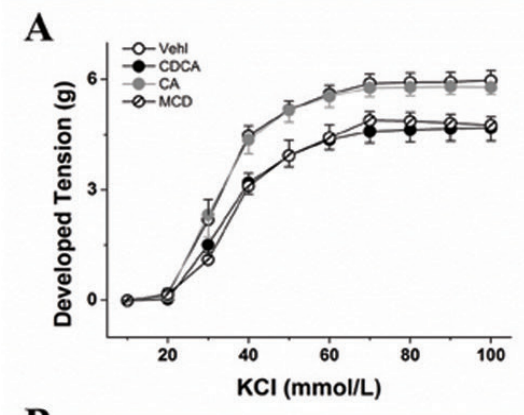

B

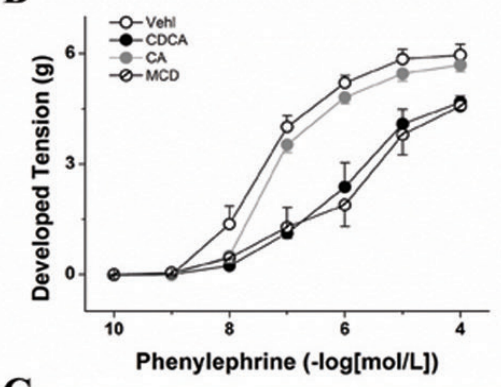

C

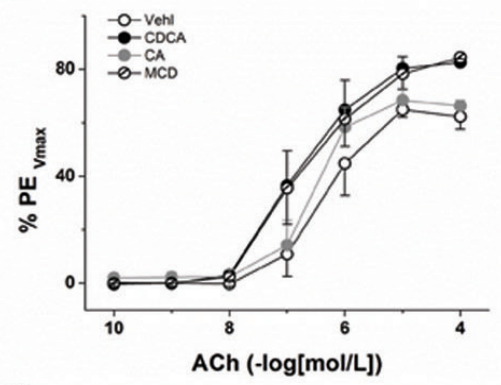

D

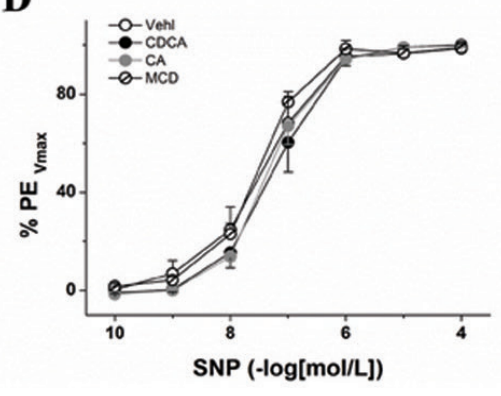

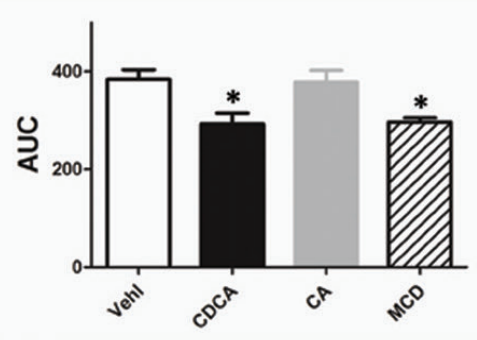
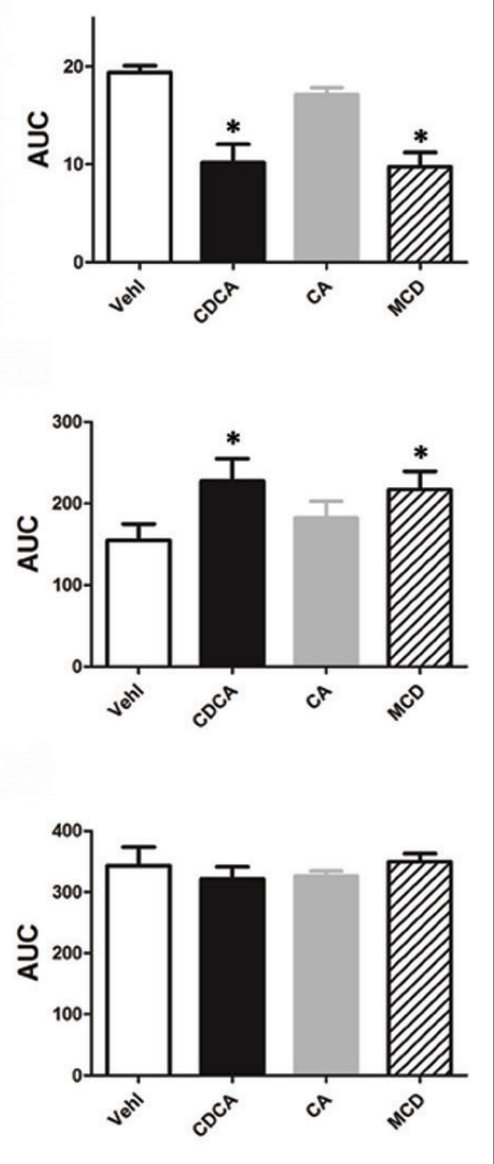

(Fig. 1D). Conversely, as compared to Vehl, in endothelium-denuded abdominal aorta, none of CDCA, MCD or CA changed the vasoconstriction elicited by either KCl or PE (Fig. 2).

\section{Effects of CDCA or CA on caveolae abundance}

The structure of caveolae on the membrane of ECs is important for integrating various signaling pathways and inducing downstream cellular events. Thus, next in the present study, TEM was conducted to examine caveolae structure in ECs of abdominal aorta. Normally, caveolae are distinguished by their characteristic size (50-100 nm), flask-like shape, and location on or in juxtaposition to the plasma membrane. As shown by original and magnified images as well as summarized data in Fig. 3, a plethora of caveolae was found on ECs of Vehltreated abdominal aorta. CDCA and MCD but not CA significantly reduced the quantity of caveolae $(P<0.05)$ which could be restored by $\mathrm{Chl}$ supplementation. However, Chl did not influence caveolae content in CA-treated vessels. 
Fig. 2. Effects of CDCA, CA or MCD on vasoconstrictive responses of abdominal aorta without endothelium. Concentration-dependent responses of endothelium-denuded abdominal aorta to $\mathrm{KCl}(\mathrm{A})$ or $\mathrm{PE}$ (B) in the presence of Vehl, CDCA, CA or MCD and AUC of each test. Values are means \pm SE. $n=8,{ }^{*} P<0.05$ vs. Vehl.

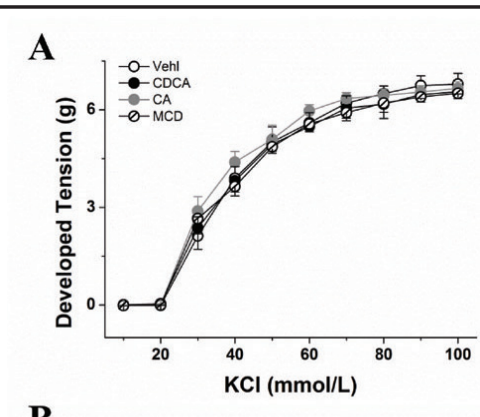

B

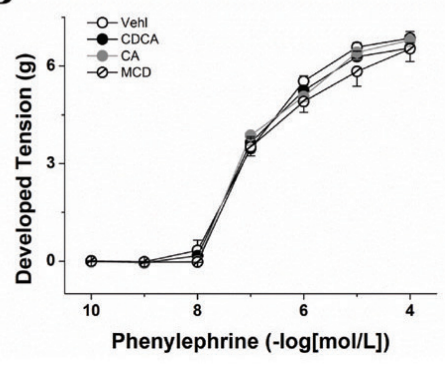

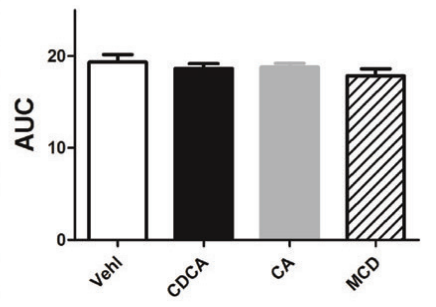

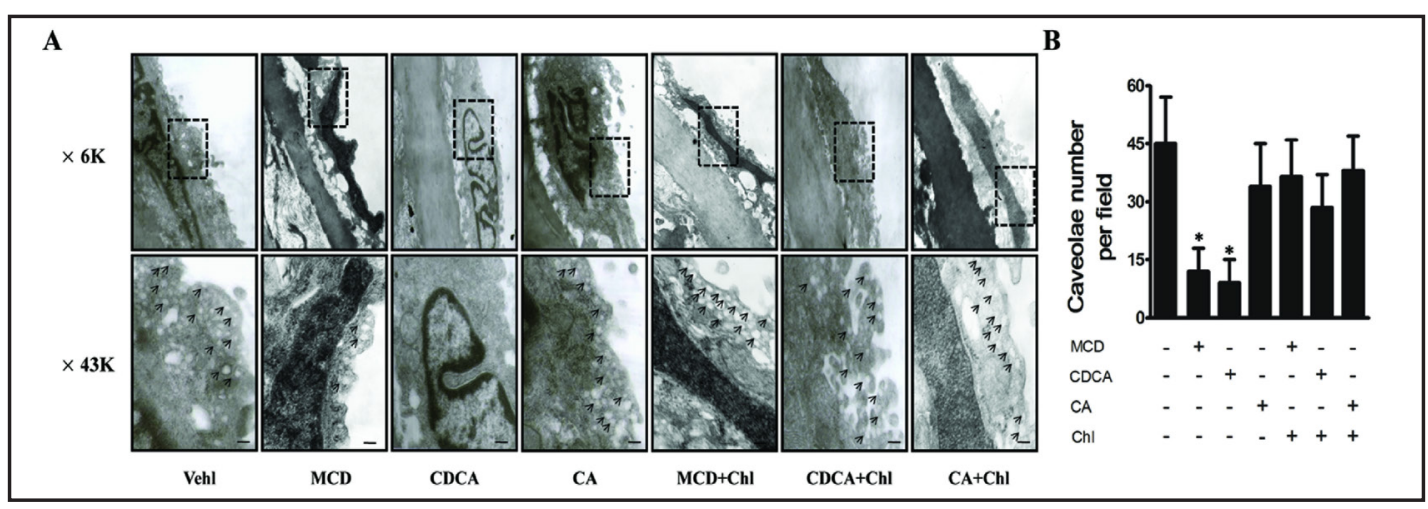

Fig. 3. Effects of MCD or bile acids (BAs) with or without cholesterol (Chl) treatment on caveolae abundance in endothelial cells (ECs) of abdominal aorta. (A) Micrograph of caveolae structure on ECs of abdominal aorta. Magnification $\times 6000$ (up layer) and $\times$ 43, 000 (low layer); scale bars, $100 \mathrm{~nm}$; black arrows indicate flask-shaped invaginations of caveolae. (B) summarized data showing average caveolae number per field. Values are means \pm SE. $n=4,{ }^{*} P<0.05$ vs. groups without treatment of CDCA, CA, MCD or Chl.

\section{Effects of CDCA or CA on eNOS expression, phosphorylation and NO production}

eNOS activity and the subsequent NO production is one of the main determinants of vasoreactivity, which is also regulated by caveolae, so we next detected the protein expression and phosphorylation of eNOS as well as NO generation in abdominal aorta. As shown by Western blot analysis in Fig. 4A, endothelium-denudation significantly reduced protein expression of total eNOS (t-eNOS) $(P<0.05)$. In endothelium-intact abdominal aorta, CDCA or MCD but not CA significantly reduced protein expression of t-eNOS and enhanced that of phosphorylated eNOS (p-eNOS) at Thr ${ }^{495}$ as compared to Vehl $(P<0.05)$ which was reversed by $\mathrm{Chl}$ incubation (Fig. 4B). However, in endothelium-denuded abdominal aorta, none of CDCA, MCD or CA had significant influence on t-eNOS or p-eNOS expression which was also unaffected by $\mathrm{Chl}$ incubation (Fig. 4C). Nitrate and nitrite content was measured to calculate NO production. In line with the eNOS alterations, in endothelium-intact abdominal aorta, CDCA or MCD but not CA significantly increased NO production as compared to Vehl (Fig. 5, $P<0.05$ ), which was restored by Chl treatment.

\section{KARGER}


A
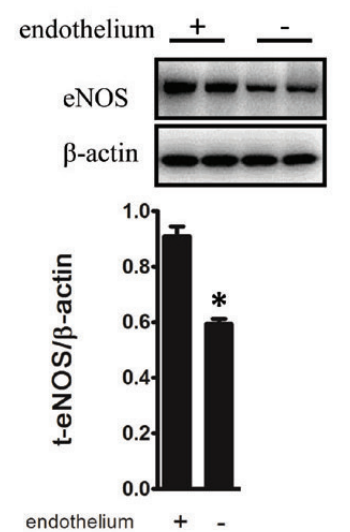

B
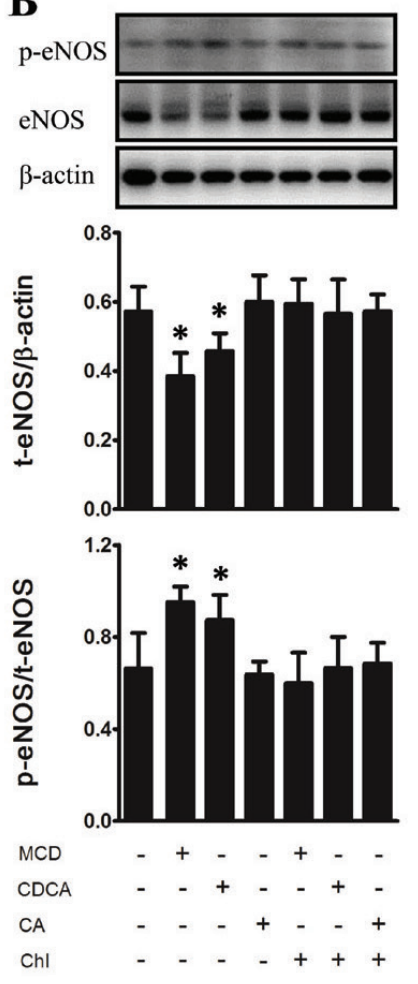

C
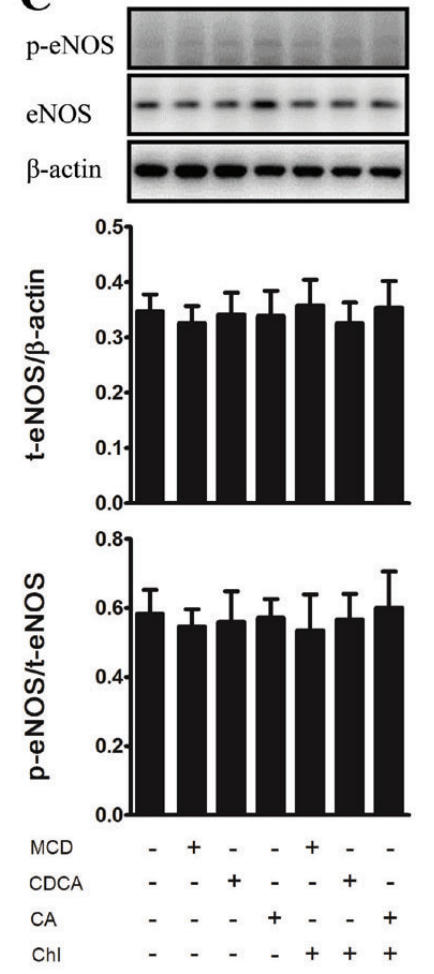

Fig. 4. Protein expression of total endothelial nitric oxide synthase (t-eNOS) and phosphorylated eNOS (p-eNOS) in endothelium-intact or -denuded abdominal aorta treated by CDCA, CA or MCD with or without Chl incubation. (A) Representative band and summarized data of t-eNOS protein expression in Western blot analysis of abdominal aorta with or without endothelium. B and C, Representative band and summarized data of t-eNOS or p-eNOS protein expression in Western blot analysis of abdominal aorta with (B) or without (C) endothelium treated by CDCA, CA or MCD in the presence of Chl incubation or not. Values are means \pm SE. $n=6,{ }^{*} P<0.05$ vs. groups without treatment of CDCA, CA, MCD or Chl.

Fig. 5. Summarized data depicting nitrate and nitrite concentration in endothelium-intact abdominal aorta treated by CDCA, CA or MCD with or without Chl incubation. Values are means \pm SE. $n=6,{ }^{*} P<0.05$ vs. groups without treatment of CDCA, CA, MCD or Chl.

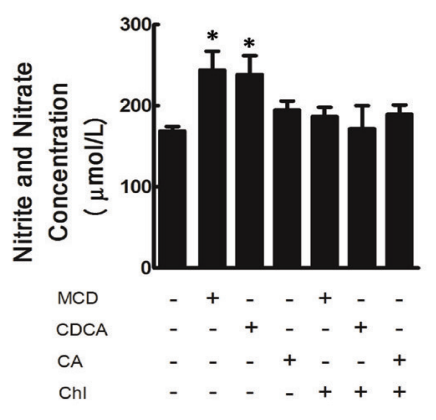

Effects of Chl on vasoactive effects of CDCA or CA

Since CDCA interfered with caveolae structure promoting eNOS phosphorylation as well as NO production remarkably, we then investigated the role of caveolae in vasorelaxtant effect of CDCA. As shown by Fig. 6A and B, with Chl incubation, CDCA, CA or MCD did not change the vasoconstriction elicited by either $\mathrm{KCl}$ or $\mathrm{PE}$ as compared to Vehl in endotheliumintact abdominal aorta. Meanwhile, in the presence of $\mathrm{Chl}$, there was no significant difference in Ach- or SNP-elicited vasodilatation among CDCA-, CA-, MCD- or Vehl-treated vessels (Fig. 6C and 6D). 
Fig. 6. Effects of $\mathrm{Chl}$ incubation on vascular responses of abdominal aorta with treatment of CDCA, CA or MCD. Concentration-dependent responses of abdominal aorta to $\mathrm{KCl}$ (A), PE (B), Ach (C) or SNP (D) in the presence of Vehl, CDCA, CA or MCD with $\mathrm{Chl}$ incubation. Values are means \pm SE. $n=8$.

\section{Discussion}

In this study, the disparate effect of hydrophobic and hydrophilicBAs on vasorelaxant responses of abdominal aorta and the mechanism involving caveolae abundance on ECs was investigated. We found CDCA but not CA reduced both $\mathrm{KCl}$ - and $\mathrm{PE}-$ elicited vasoconstriction and enhanced Ach-elicited vasodilatation which occurred with diminished caveolae structure on ECs of abdominal aorta. Eliminating caveolae with MCD exerted same effects on vasoconstriction and vasodilatation as CDCA while endothelium-denudation prevented the function of both CDCA and MCD. Disruption of

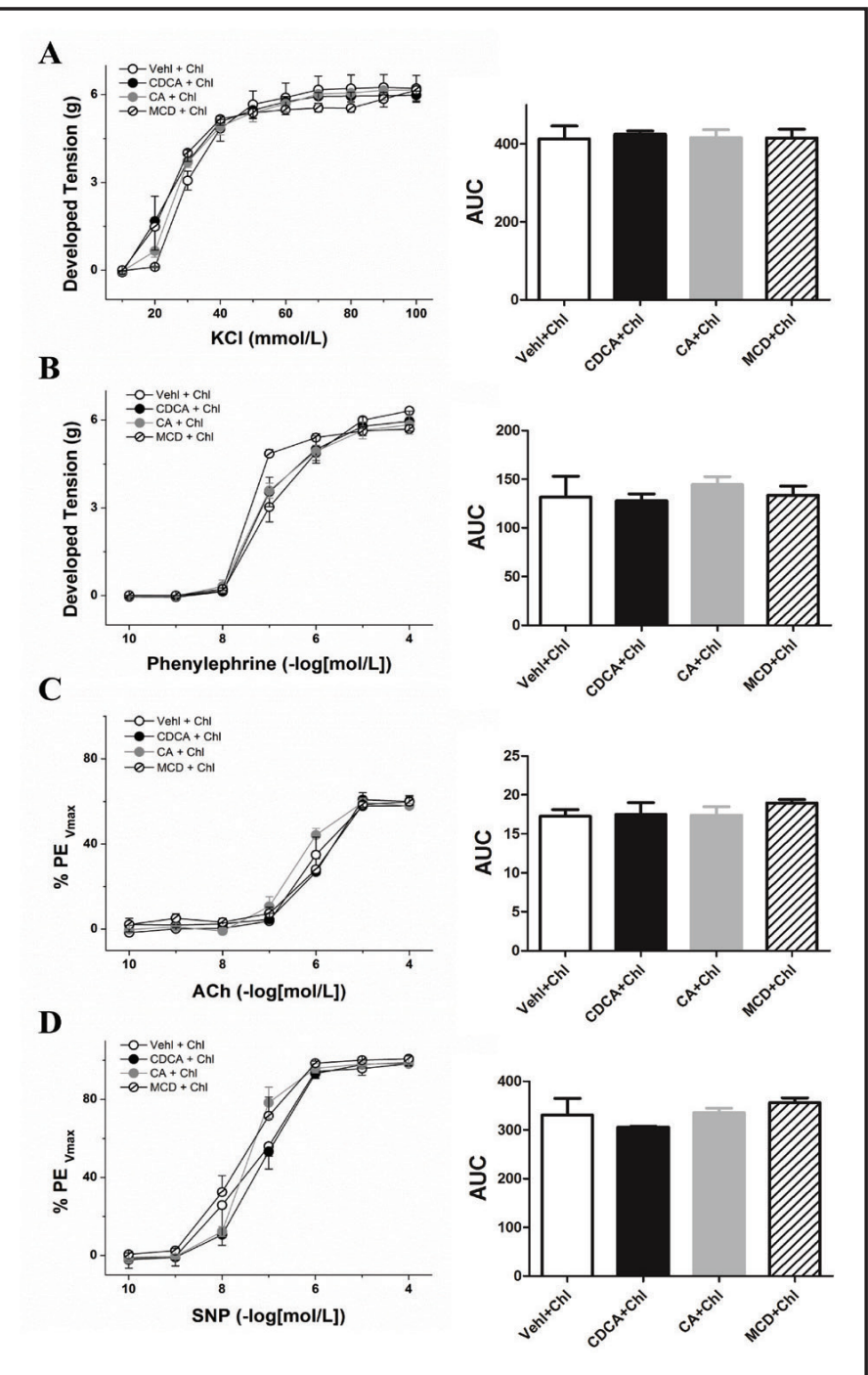

B

$\mathbf{D}$

caveolae by CDCA and MCD was accompanied by increased eNOS phosphorylation at Thr ${ }^{495}$ and enhanced NO production, both of which could be restored by Chl replenishment. Then we found $\mathrm{Chl}$ incubation completely reversed the vasorelaxtant effects of both CDCA and MCD.

BAs are amphiphilic steroids synthesized from free cholesterol in hepatocytes essential for the emulsification and solubilization of lipids in bile and the gastrointestinal tract $[1,2]$. Normally, only $5 \%$ of the biliary BAs are newly synthesized, and most of them are obtained through enterohepatic circulation [16]. Postprandial serum BAs level ranges between 2 and $10 \mu \mathrm{mol} / \mathrm{L}$, which is 6 times lower than that in the portal circulation [17]. However, significant spillover of BAs from portal to systemic circulation usually occurs in hepatobiliary disorders, such as obstructive cholestasis, progressive familial intrahepatic cholestasis (PFIC), hepatic cirrhosis, and intrahepatic cholestasis during pregnancy $[3,18,19]$. In these situations, the concentration of BAs in serum would be boosted even exceeding $200 \mu \mathrm{mol} / \mathrm{L}[1,16]$, which is a potent stimulus to ECs and vascular smooth muscle cells (VSMCs) and could partly explain vascular dysfunction or system hypotension in cirrhosis or other conditions associated with elevated serum BAs [20,21].

Copious evidences have demonstrated the vasorelaxant ability of high level BAs and the lipid solubility dependence of the response. Generally, hydrophobic BAs exert greater relaxant effects than hydrophilic ones because they are more efficient to change the composition of cell membrane [6]. Hydrophobicity of BAs is determined by the status of ionization, 


\section{Cellular Physiology Cell Physiol Biochem 2017;42:1013-1024 \\ \begin{tabular}{l|l} 
DOI: 10.1159/000478683 & O 2017 The Author(s). Published by S. Karger AG, Basel \\
www.karger.com/cpb
\end{tabular}}

Wang et al.: Caveolae Counteracts Vasorelaxant Effects of CDCA

orientation, position and number of hydroxyl groups, and the presence of the side chain ester [6]. Normally, conjugation and presence of hydroxyl groups decrease hydrophobicity of BAs. Thus, dihydroxy BAs such as CDCA and DCA retains more vasoactive effects than trihydroxy BAs such as CA and amide conjugates of BAs [22, 23]. In our work, CDCA and CA were adopted to represent hydrophobic and hydrophilic BA, respectively. The concentration of $100 \mu \mathrm{mol} / \mathrm{L}$ we used was comparable to the level of serum BAs in cholestatic diseases. In the test of isometric force recording, we found CDCA but not CA significantly decreased vasoconstriction to both $\mathrm{KCl}$ and $\mathrm{PE}$ in abdominal aorta which was akin to the previous studies $[5,6]$.

In the present work, we noticed the Ach-elicited endothelium-dependent but not the SNP-elicited endothelium-independent vasodilatation was enhanced by CDCA, while CA did not show the same effect, indicating the ECs function of abdominal aorta was improved by hydrophobic BAs. As NO produced by ECs determines the intensity of vasoconstriction, the upregulation of ECs function might lead to the negative inotropism effects of CDCA. The deduction was further proved by our finding that CDCA also induced changes in vasoconstriction of abdominal aorta elicited by both $\mathrm{KCl}$ and $\mathrm{PE}$ and could be completely diminished by endothelium-denudation. This result was in accordance with reports showing hydrophobic BAs increased both expression and activity of eNOS, accelerating NO generation in mesenteric arteries [24] and cultured ECs [25, 26] and inhibition of eNOS activity attenuated deoxycholyltaurine-induced vasodilatation in rat and mouse aorta [27].

The mechanisms underlying the vasorelaxant effect of BAs are still incompletely understood. Multifaceted processes have been proposed including lower fluidity of cell membrane along with decreased affinity of $\alpha 1$-adrenoceptors [6], activation of muscarinic M3R- or M2R-dependent molecular signaling [1, 27], increased BK ${ }_{\mathrm{Ca}}$ activity $[8,28]$, inhibition of $\mathrm{Ca}^{2+}$ entry and $\mathrm{Na}^{+} / \mathrm{Ca}^{2+}$ exchanger (NCX) current $[16,29]$, activated TGR5 also known as $\mathrm{G}$ protein-coupled bile acid receptor 1 (GPBAR1) [30] and depressed vasoconstrictive response to endothelin-1 (ET-1) [24].

Caveolae are flask-shaped invaginations with diameter of 50-100 nm on the plasma membrane enriched of cholesterol and sphingolipids [9]. Caveolin is the main structural protein of caveolae and could be divided into three subtypes as Cav-1, caveolin-2 and caveolin-3 [31]. Among them, Cav-1 is most prominently expressed in vascular ECs through which eNOS is targeted to caveolae by cotranslational $N$-myristoylation and posttranslational palmitoylation [11]. Cav-1 interacts with eNOS via the scaffolding domain resulting in the inhibition of eNOS activity and NO production [10,32,33], so we next tried to elucidate the role of caveolae in the changes of eNOS/NO pathway and vasorelaxation induced by CDCA.

As caveolae is 10 -fold more enriched of $\mathrm{Chl}$ than the bulk plasma membrane and the integrity of caveolae depends a lot on adequate Chl content, Chl adjustment has always been the choice for the modification of caveolae content [15]. MCD, which selectively entraps $\mathrm{Chl}$ rather than other membrane lipids, could effectively remove Chl from membranes and has been employed extensively to reduce the amount of caveolae $[13,15]$. Inversely, Chl supplementation is always undertaken to increase caveolae content [15, 34]. We compared the effect of BAs on vasoreactivity of abdominal aorta with that of MCD and demonstrated that, similar to CDCA, MCD also decreased vasoconstriction and increased endotheliumdependent vasodilatation, suggesting the effect of CDCA might be achieved though the caveolae disruption on ECs of abdominal aorta.

We then tested how the BAs altered the formation of caveolae and the result showed that there was plethora of caveolae on membranes of ECs in abdominal aorta, which was significantly reduced by CDCA as well as MCD but not CA and could be completely restored by Chl replenishment. It is well acknowledged that BAs solubilize membranes through clearing Chl and phospholipid which might be the reason why CDCA reduced caveolae prominently $[6,35]$. Although there was report showing millimolar but not micromolar level of BAs is concentrated enough to induce changes of membrane fluidity and reduction of Chl and phospholipids [6], our result suggests $100 \mu \mathrm{mol} / \mathrm{L}$ of CDCA could also imbalance Chl metabolism on cell membrane and deplete caveolae structure there. Moreover, along with 
the decline of caveolae on ECs, we found eNOS phosphorylation and NO production were boosted by CDCA and MCD but not CA which was absent in endothelium-denuded artery and could be fully reversed by Chl replenishment, further proving the negative effects of caveolae structure on eNOS bioactivity in ECs. Simultaneously, after the caveolae was restored by $\mathrm{Chl}$, the aberrant vasoconstriction and vasodilatation of abdominal aorta induced by CDCA and MCD was also corrected. Thus, we demonstrate that CDCA, a hydrophobic BA, elicited a vasorelaxant effect on abdominal aorta via disrupting caveolae structure of ECs which promotes the activation of eNOS and subsequent NO production. Our result is in consistent with previous studies showing upregulation of caveolae was induced by Ang II infusion in ApoE (-/-) mice which mediated reduced NO bioavailability and endothelial dysfunction in aorta $[36,37]$. Besides, all these effects were reversed by disruption of caveolae by MCD. However, we also noticed the report showing caveolae depletion or Cav-1 gene deficiency reduced shear stress-mediated vasodilatation in small coronary arteries of mice for the generation of vasodilators including NO, prostaglandins and epoxydicosatrienoic acids was inhibited [38]. The distinct outcomes suggest the role of caveolae in vasoreactivity might be complicated varying among different species, arteries or stimuli. Moreover, based on the report, we speculate that by diminishing caveolae on ECs, apart from systemic hypotension, CDCA might exert detrimental functions on coronary regulation which serves as another mechanism underlying the cardiovascular disorder in cholestasis.

The role of caveolae in transcytosis, endocytosis, Chl transport and signal transduction has long been known [10]. For example, the uptake of folate receptor, cholera, tetanus toxins, and alkaline phosphatase relies largely on caveolae [10,39]. Simian virus 40 (SV40) and certain strains of Escherichia coli are endocytosed via receptors resident in caveolae. Besides, Cav-1 can help deliver $\mathrm{Chl}$ from the endoplasmic reticulum (ER) to caveolae on plasma membrane $[10,39,40]$. At this juncture, Chl can either be transferred to extracellular lipoproteins or be siphoned into the bulk plasma [41-44]. So the depletion of caveolae structure would reduce the content of $\mathrm{Chl}$ on cell membrane which might further suppress the caveolae abundance there. In view of the diversified use of caveolae, we predict that CDCA may possess much more probability in structural or functional remodeling of cardiovascular system.

In summary, we demonstrated that CDCA but not CA induced vasorelaxation of abdominal aorta by diminishing the caveolae structure on ECs which promotes eNOS phosphorylation and NO production. Our present study proposes a new mechanism underlying the vasorelaxant effect of hydrophobic BAs which might help shed light on manifestation of cholestatic diseases in cardiovascular system.

\section{Funding}

This study was supported by National Natural Science Foundation of China (Grant No. 31071045, 81401550).

\section{Acknowledgements}

We would like to thank all other staff from the Department of Aerospace Physiology, and Department of Aerospace Hygiene, Fourth Military Medical University for their help with the animals and equipment maintenances.

\section{Disclosure Statement}

No conflict of interests. 


\section{Cellular Physiology Cell Physiol Biochem 2017;42:1013-1024 \begin{tabular}{l|l} 
and Biochemistry Published online: June 28, 2017 & $\begin{array}{l}\text { (C) } 2017 \text { The Author(s). Published by S. Karger AG, Basel } \\
\text { www.karger.com/cpb }\end{array}$
\end{tabular}}

Wang et al.: Caveolae Counteracts Vasorelaxant Effects of CDCA

\section{References}

$\rightarrow 1$ Li T, Chiang JY: Bile acids as metabolic regulators. Curr Opin Gastroenterol 2015;31:159-165.

2 Li T, Chiang JY: Bile acid signaling in metabolic disease and drug therapy. Pharmacol Rev 2014;66:948-983.

-3 Qin P, Tang X, Elloso MM, Harnish DC: Bile acids induce adhesion molecule expression in endothelial cells through activation of reactive oxygen species, NF-kappaB, and p38. Am J Physiol Heart Circ Physiol 2006;291:H741-H747.

4 Pazzi P, Morsiani E, Vilei MT, Granato A, Rozga J, Demetriou AA, Muraca M: Serum bile acids in patients with liver failure supported with a bioartificial liver. Aliment Pharmacol Ther 2002;16:1547-1554.

-5 Estrela HF, Damasio ES, Fonseca EK, Bergamaschi CT, Campos RR: Differential sympathetic vasomotor activation induced by liver cirrhosis in rats. Plos One 2016;11:e152512.

-6 Ljubuncic P, Said O, Ehrlich Y, Meddings JB, Shaffer EA, Bomzon A: On the in vitro vasoactivity of bile acids. Br J Pharmacol 2000;131:387-398.

7 Kida T, Tsubosaka Y, Hori M, Ozaki H, Murata T: Bile acid receptor TGR5 agonism induces NO production and reduces monocyte adhesion in vascular endothelial cells. Arterioscler Thromb Vasc Biol 2013;33:1663-1669.

8 Bukiya AN, Liu J, Toro L, Dopico AM: Beta1 (KCNMB1) subunits mediate lithocholate activation of largeconductance $\mathrm{Ca} 2+$-activated $\mathrm{K}+$ channels and dilation in small, resistance-size arteries. Mol Pharmacol 2007;72:359-369.

-9 Parton RG, Simons K: The multiple faces of caveolae. Nat Rev Mol Cell Biol 2007;8:185-194.

10 Razani B, Lisanti MP: Caveolins and caveolae: Molecular and functional relationships. Exp Cell Res 2001;271:36-44.

11 Shaul PW: Endothelial nitric oxide synthase, caveolae and the development of atherosclerosis. J Physiol 2003;547:21-33.

12 Shi F, Zhao TZ, Wang YC, Cao XS, Yang CB, Gao Y, Li CF, Zhao JD, Zhang S, Sun XQ: The impact of simulated weightlessness on Endothelium-Dependent angiogenesis and the role of Caveolae/Caveolin-1. Cell Physiol Biochem 2016;38:502-513.

13 Dreja K, Voldstedlund M, Vinten J, Tranum-Jensen J, Hellstrand P, Sward K: Cholesterol depletion disrupts caveolae and differentially impairs agonist-induced arterial contraction. Arterioscler Thromb Vasc Biol 2002;22:1267-1272.

14 Schubert R, Schmidt KH: Structural changes in vesicle membranes and mixed micelles of various lipid compositions after binding of different bile salts. Biochemistry-US 1988;27:8787-8794.

15 Wang Z, Bai Y, Yu J, Liu H, Cheng Y, Liu Y, Xie X, Ma J, Bao J: Caveolae regulate vasoconstriction of conduit arteries to angiotensin II in hindlimb unweighted rats. J Physiol 2015;593:4561-4574.

-16 Zhu J, Dong X, Liu Q, Wu C, Wang Q Long Z, Li L: Hydrophobic bile acids relax rat detrusor contraction via inhibiting the opening of the $\mathrm{Na}(+) / \mathrm{Ca}(2)(+)$ exchanger. Sci Rep 2016;6:21358.

17 Linnet K: Postprandial plasma concentrations of glycine and taurine conjugated bile acids in healthy subjects. Gut 1983;24:249-252.

18 Glantz A, Marschall HU, Mattsson LA: Intrahepatic cholestasis of pregnancy: Relationships between bile acid levels and fetal complication rates. Hepatology 2004;40:467-474.

-19 Azer SA, Coverdale SA, Byth K, Farrell GC, Stacey NH: Sequential changes in serum levels of individual bile acids in patients with chronic cholestatic liver disease. J Gastroenterol Hepatol 1996;11:208-215.

20 Song JG, Cao YF, Sun YM, Ge YH, Xu XW, Yang LQ, Liu ZQ, Song SL, Yu WF: Baroreflex sensitivity is impaired in patients with obstructive jaundice. Anesthesiology 2009;111:561-565.

-21 Sanyal AJ, Bosch J, Blei A, Arroyo V: Portal hypertension and its complications. Gastroenterology 2008;134:1715-1728.

22 Heuman DM: Quantitative estimation of the hydrophilic-hydrophobic balance of mixed bile salt solutions. J Lipid Res 1989;30:719-730.

23 Roda A, Minutello A, Angellotti MA, Fini A: Bile acid structure-activity relationship: Evaluation of bile acid lipophilicity using 1-octanol/water partition coefficient and reverse phase HPLC. J Lipid Res 1990;31:1433-1443.

24 Li C, Li J, Weng X, Lan X, Chi X: Farnesoid X receptor agonist CDCA reduces blood pressure and regulates vascular tone in spontaneously hypertensive rats. J Am Soc Hypertens 2015;9:507-516. 


\section{Cellular Physiology Cell Physiol Biochem 2017;42:1013-1024 \begin{tabular}{l|l} 
DOI: 10.1159/000478683 & and Biochemistry \\
Published online: June 28, 2017 & $\begin{array}{l}\text { 2017 The Author(s). Published by S. Karger AG, Basel } \\
\text { www.karger.com/cpb }\end{array}$ \\
\hline
\end{tabular}}

Wang et al.: Caveolae Counteracts Vasorelaxant Effects of CDCA

25 Nakajima T, Okuda Y, Chisaki K, Shin WS, Iwasawa K, Morita T, Matsumoto A, Suzuki JI, Suzuki S, Yamada N, Toyo-Oka T, Nagai R, Omata M: Bile acids increase intracellular $\mathrm{Ca}(2+)$ concentration and nitric oxide production in vascular endothelial cells. Br J Pharmacol 2000;130:1457-1467.

-26 Keitel V, Reinehr R, Gatsios P, Rupprecht C, Gorg B, Selbach O, Haussinger D, Kubitz R: The G-protein coupled bile salt receptor TGR5 is expressed in liver sinusoidal endothelial cells. Hepatology 2007;45:695704.

27 Khurana S, Yamada M, Wess J, Kennedy RH, Raufman JP: Deoxycholyltaurine-induced vasodilation of rodent aorta is nitric oxide- and muscarinic M(3) receptor-dependent. Eur J Pharmacol 2005;517:103-110.

-28 Dopico AM, Walsh JJ, Singer JJ: Natural bile acids and synthetic analogues modulate large conductance Ca2+-activated K+ (BKCa) channel activity in smooth muscle cells. J Gen Physiol 2002;119:251-273.

29 Khurana S, Raina H, Pappas V, Raufman JP, Pallone TL: Effects of deoxycholylglycine, a conjugated secondary bile acid, on myogenic tone and agonist-induced contraction in rat resistance arteries. Plos One 2012; 7:e32006.

-30 Keitel V, Cupisti K, Ullmer C, Knoefel WT, Kubitz R, Haussinger D: The membrane-bound bile acid receptor TGR5 is localized in the epithelium of human gallbladders. Hepatology 2009;50:861-870.

-31 Kim D, Kim H, Koo JS: Expression of caveolin-1, caveolin-2 and caveolin-3 in thyroid cancer and stroma. Pathobiology 2012;79:1-10.

32 Goligorsky MS, Li H, Brodsky S, Chen J: Relationships between caveolae and eNOS: Everything in proximity and the proximity of everything. Am J Physiol Renal Physiol 2002;283:F1-F10.

-33 Albrecht EW, Stegeman CA, Heeringa P, Henning RH, van Goor H: Protective role of endothelial nitric oxide synthase. J Pathol 2003;199:8-17.

-34 Cohen AW, Hnasko R, Schubert W, Lisanti MP: Role of caveolae and caveolins in health and disease. Physiol Rev 2004;84:1341-1379.

-35 Akare S, Martinez JD: Bile acid induces hydrophobicity-dependent membrane alterations. Biochim Biophys Acta 2005;1735:59-67.

36 Forrester SJ, Elliott KJ, Kawai T, Obama T, Boyer MJ, Preston KJ, Yan Z, Eguchi S, Rizzo V: Caveolin-1 deletion prevents hypertensive vascular remodeling induced by angiotensin II. Hypertension 2017;69:79-86.

37 Seto SW, Krishna SM, Yu H, Liu D, Khosla S, Golledge J: Impaired acetylcholine-induced endotheliumdependent aortic relaxation by caveolin-1 in angiotensin II-infused apolipoprotein-E (ApoE-/-) knockout mice. Plos One 2013;8:e58481.

-38 Chai Q, Wang XL, Zeldin DC, Lee HC: Role of caveolae in shear stress-mediated endothelium-dependent dilation in coronary arteries. Cardiovasc Res 2013;100:151-159.

39 Nabi IR, Le PU: Caveolae/raft-dependent endocytosis. J CELL BIOL 2003;161:673-677.

-40 Ding W, Wang B, Zhang M, Gu Y: Involvement of endoplasmic reticulum stress in uremic cardiomyopathy: Protective effects of tauroursodeoxycholic acid. Cell Physiol Biochem 2016;38:141-152.

-41 Smart EJ, Ying YS, Conrad PA, Anderson RG: Caveolin moves from caveolae to the Golgi apparatus in response to cholesterol oxidation. J Cell Biol 1994;127:1185-1197.

-42 Smart EJ, Ying Y, Donzell WC, Anderson RG: A role for caveolin in transport of cholesterol from endoplasmic reticulum to plasma membrane. J Biol Chem 1996;271:29427-29435.

-43 Sommerfeld A, Reinehr R, Haussinger D: Tauroursodeoxycholate Protects Rat Hepatocytes from Bile AcidInduced Apoptosis via beta1-Integrin- and Protein Kinase A-Dependent Mechanisms. Cell Physiol Biochem 2015;36:866-883.

44 Grothe J, Riethmuller J, Tschurtz SM, Raith M, Pynn CJ, Stoll D, Bernhard W: Plasma phosphatidylcholine alterations in cystic fibrosis patients: Impaired metabolism and correlation with lung function and inflammation. Cell Physiol Biochem 2015;35:1437-1453. 